Cinémas

Revue d'études cinématographiques

Journal of Film Studies

\title{
WARREN, Paul. Le Secret du star system américain. Une stratégie du regard. Montréal : L’Hexagone, 1989, 204 p.
}

\section{Denise Pérusse}

Volume 1, numéro 1-2, automne 1990

URI : https://id.erudit.org/iderudit/1000997ar

DOI : https://doi.org/10.7202/1000997ar

Aller au sommaire du numéro

Éditeur(s)

Cinémas

ISSN

1181-6945 (imprimé)

1705-6500 (numérique)

Découvrir la revue

Citer ce compte rendu

Pérusse, D. (1990). Compte rendu de [WARREN, Paul. Le Secret du star system américain. Une stratégie du regard. Montréal : L’Hexagone, 1989, 204 p.]

Cinémas, 1(1-2), 146-152. https://doi.org/10.7202/1000997ar d'utilisation que vous pouvez consulter en ligne.

https://apropos.erudit.org/fr/usagers/politique-dutilisation/ 
WARREN, Paul. Le Secret du star system américain. Une stratégie du regard. Montréal: L'Hexagone, 1989, 204 p.

Le Secret du star system américain constitue le fruit d'une réflexion de plusieurs années de recherches effectuées par Paul Warren sur le cinéma hollywoodien. Cet ouvrage-synthèse reprend pour une large part des questions qu'il a traitées dans des articles publiés dans diverses revues, qu'il a remaniés et enrichis. L'auteur fait preuve d'un talent remarquable de conteur dans son souci de démonter les ressorts du cinéma hollywoodien, énorme machine voyeuriste reposant essentiellement, selon l'auteur, sur une technique simple mais ô combien efficace: le reaction shot. Paul Warren s'est d'ailleurs attiré pour cet essai le prix de l'Union des éditeurs de langue française.

Le Secret du star system américain s'avère un ouvrage de base pour comprendre le cinéma américain. Malgré les multiples dérives qui sillonnent le parcours de l'auteur, l'argumentation de cet essai synthèse se développe clairement et logiquement. Paul Warren prend bien soin de tisser ses dérives par un fil narratif commun: le jeu cinématographique du reaction shot. Les chapitres sont bien délimités et la présentation des idées, cohérente.

Selon l'hypothèse centrale de Warren, la technique du reaction shot, à savoir le regard de réaction des protagonistes de l'écran, constitue le pilier central qui détermine les autres regards (le regard du réalisateur et celui des spectateurs sur l'écran) et déclenche le processus fictionnel. L'auteur est fasciné par cette structure qui a fait l'âge d'or du cinéma américain et, pour déployer sa thèse principale, il fait appel à un corpus varié, voire même éclaté. Les exemples sont puisés tant du côté de la boîte à images que de celui du grand écran. Les hommes politiques, les prédicateurs, les stars se côtoient, les références à divers courants cinématographiques (réalisme soviétique, expressionnisme allemand, etc.) se multiplient; des réalisateurs aussi différents que Godard, Murnau, Eisenstein, Capra, et j'en passe, sont conviés à la même enseigne, le «coulissage» d'un genre à l'autre est instauré en principe. Warren s'appuie également sur bon nombre d'exemples puisés dans le corpus américain: San Francisco, Good Morning Vietnam, la série Rocky, Airport, Taxi Driver, etc. De même, il alimente son essai en glanant dans quelques-unes de ses expériences dans le milieu cinématographique américain. Le but visé est toujours de montrer comment cette technique cinématographique qu'est le reaction shot est fondamentale, commande la structure des séquences, tout en contribuant à la vraisemblance comme au naturalisme de l'image cinématogra- 
phique, tout en appellant un type de jeu spécifique, soit celui orchestré par l'Actor's Studio.

Premier principe à retenir: la suture opérée par le duo champ/hors champ. Le reaction shot sert avant tout à déplacer le regard du spectateur du champ vers le hors champ - tout ce qui n'est pas cadré (sur les côtés, en dessus, en dessous, en avant, derrière l'image perceptible) par l'image - , un hors champ qui peut surgir à tout moment et que le spectateur appelle de tout cœur. Le hors champ devient une véritable «usine de reaction shots», et lorsqu'il vient balayer l'ombre d'un instant ce qui était dans le champ, il correspond en toutes lettres à ce que le spectateur avait anticipé. Le hors champ est donc la seconde nature du champ cinématographique, c'est lui qui «sécrète» le champ, c'est là où se love le regard du regardant et par extension le regard spectatoriel. Comme le dit si bien Metz, et que reprend l'auteur à juste titre, «le hors champ est le propre (le lieu propre) du spectateur» (p. 24). Et c'est dans ce transfert du champ vers le hors champ que se fait la suture, que la technique se fait invisible.

Deuxième principe de base: «la vedettarisation par le reaction shot». Si le reaction shot constitue le lieu privilégié de l'espace spectatoriel, cette technique demeure l'une des plus fameuses trouvailles par Hollywood pour vedettariser les stars. Ce sont les multiples regards des regardants, des protagonistes et par extension des spectateurs, "couvant», balayant la star qui rendent possible sa vedettarisation. Pour affûter sa thèse, l'auteur puise quelques exemples tant dans l'univers cinématographique que télévisuel et même dans la réalité américaine. Marilyn Monroe, Ronald Reagan et Jimmy Swaggart passent ainsi dans la moulinette du reaction shot.

Dans son souci de déconstruire le star-system et le modèle hollywoodien, l'auteur fait, nous l'avons souligné, de nombreux détours pour montrer que l'édifice repose sur une technique «fascinante» mais «fascisante», relais pernicieux qui installe confortablement les grands mythes américains que sont l'entraide, la conquête de l'Ouest, le triomphe de la civilisation sur la sauvagerie, le dépassement vers la frontière, l'élection divine, l'individualisme triomphant. Les pérégrinations de l'auteur nous conduisent notamment au cœur d'une œuvre de la période nazie, à savoir le célèbre Triomphe de la volonté de Leni Riefenstahl. Triomphe de la volonté certes, clame l'auteur, mais surtout et avant tout triomphe du reaction shot: «L'Allemagne nazie toute entière est réduite à une réaction unique et monstrueuse à l'acteur unique, Adolf Hitler» (p. 13). Ce saut dans le cinéma du IIIe Reich sert de prétexte pour scruter les films de fiction américains et montrer comment le reaction shot oblige une réflexion sur la notion de réalisme qui caractérise le cinéma hollywoodien. 
L'auteur puise également de nombreux exemples au sein de l'univers télévisuel dans le but de montrer comment la boîte à images a récupéré les structures de base du septième art et permis de consolider, de «blinder» le modèle filmique dominant, le cinéma narratif représentatif, qui s'appuie sur une triple trajectoire des regards, sur des croisements de regards. La plume alerte de l'auteur décortique les performances du célèbre animateur du Tonight Show, Johnny Carson, et une figure de proue de l'église «électronique», Jimmy Swaggart. Qu'il parle du déclencheur des secousses extatiques ou encore de la star de la télévision américaine, le succès du «performeur», selon Warren, tient avant tout à sa capacité de cristalliser les réactions de tous: celles de la caméra, celles des invités, celle de la salle et celle, en fin de parcours et ultimement recherchée, des téléspectateurs: "Il met en place, en scène, au premier rang et en gros plan, mon propre comportement. Il l'avalise, lui donne valeur et en garantit l'authenticité» (p. 20). Le visage du performeur devient en quelque sorte le miroir où se reflète le téléspectateur au même titre - pour faire une analogie cavalière avec l'univers de Daniel Pennac (voir La Fée Carabine et La Petite Marchande de prose - - que Benjamin Malaussène, des éditions Taillion, devient le bouc émissaire, le creuset des réactions de tous ces auteurs ratés réduits à rester dans l'ombre. Autrement dit, le prédicateur Swaggart, par exemple, n'a pu percer l'écran qu'à cause de la force du reaction shot. Les quelques plans d'auditeurs qui ponctuent le discours de Swaggart ne font que renforcer, conforter, mettre en valeur le discours et l'image du télévangéliste. Pour parler de ce vaet-vient ou de ce duo regardant/regardé, l'auteur parle même d'«effet yo-yo»: «La gestuelle et la mimique du prédicateur ne lancent la parole sur les visages du public que pour la ramener à ellemême démesurément amplifiée. Le comportement et le discours se renforcent l'un l'autre en s'alimentant constamment aux réactions du public» (p. 34-35).

Si la technique du reaction shot contribue sans nul doute au succès d'un prédicateur comme Swaggart - mais aussi de Billy Graham, de Jimmy Bakker - il ne faudrait pas toutefois opter pour un verdict réductionniste. Cette technique n'explique pas tout. Si ce type de discours obtient du succès, c'est d'abord qu'il existe un auditoire pour ce type de discours, et qu'ensuite la façon dont il est livré respecte la syntaxe télévisuelle. Si l'obscurantisme que propagent et préconisent les télévangélistes dans leur croisade contre le Mal rejoint la sensibilité d'un auditoire, c'est qu'il émerge et prend racine à la fois dans une terre accueillante. Dans cette Amérique où les extrêmes se côtoient, un citoyen sur deux exige le maintien de la séparation totale de l'Église et de l'État. Mais du même souffle, presque les trois quarts des Américains exigent d'un président qu'il ait une 
foi sincère. En fait, la religion chrétienne a une emprise considérable sur cette population bigarrée qui habite le pays se targuant d'être le plus démocratique du monde. Bref, les discours enflammés d'un Swaggart, Bakker et al. rejoignent et galvanisent un état d'esprit réceptif et prédisposé ${ }^{1}$. Par ailleurs, le respect du médium utilisé contribue également au succès des télévangélistes. Les émissions de Jim Bakker par exemple sont construites sur le modèle de "The Merv Griffin Show...". Le tournage des émissions est toujours conçu à partir d'une mise en scène précise, ce qui asservit le message au contenant. Cela donne une émission qui tient souvent davantage du divertissement (show) que de l'enchantement, du profane que du sacré. Par ailleurs, Warren vise juste lorsqu'il déclare que la télévision comme le cinéma carbure au vedettariat. L'identification du téléspectateur à un Jimmy Bakker ou à un Billy Graham est si prononcée que Dieu lui-même devient une sorte d'accessoire. Comme l'indique Neil Postman, «une des caractéristiques majeures de la télévision est qu'elle met des personnalités dans nos cœurs et non pas des abstractions dans nos têtes» (p. 165). Ainsi, face à un télévangéliste comme Swaggart, se développe une idolâtrie qui n'est pas sans rappeler celle que des admirateurs ont pu développer pour leurs stars favorites ${ }^{2}$.

Warren se sert également d'un autre exemple plus «local», tiré cette fois de la télévision québécoise, à savoir la série «Lance et compte» pour discuter du pouvoir du reaction shot. Cette fois-ci l'argumentation de Warren n'est pas centrée sur la technique du reaction shot comme moteur de la vedettarisation du héros principal - même si elle est implicite - mais plutôt comme relais puissant dans le documentaire pour faire surgir le fictionnel. «Le système du reaction shot doit se conformer aux lois du réalisme s'il veut emporter l'adhésion du spectateur.» En effet, dans le chapitre consacré à l'analyse de la série télévisuelle, Warren s'interroge sur l'hyperréalisme de «Lance et compte» en s'attardant particulièrement aux rapports qu'entretiennent documentaire et fiction dans cette série. L'intégration du documentaire se vérifie par les références à l'univers du sport et par le traitement formel des émissions. L'alternance créée par le montage entre les acteurs/personnages fictifs et les figurants/personnages réels lors d'une partie de hockey est représentative du chevauchement entre le documentaire et la fiction. Les gros plans des acteurs et les plans d'ensemble des figurants intègrent la «subjectivité» de la fiction et l'«objectivité» du documentaire. C'est ce système de «coulissage» d'un genre à l'autre qui crée l'effet de réalité: le spectateur s'identifie aux personnages de la série et se retrouve dans la foule anonyme et les lieux connus. Il intègre la réalité documentaire et fictionnelle dans le même plan. En bref, la force de frappe de la série télévisuelle, c'est d'avoir visé juste 
dans le net, soit "[d'] ancrer le filmique dans la topographie culturelle, dans les " lieux communs" de la nation, et [d'] en faire surgir le fictionnel» (p. 133). En clair, la série est populaire notamment parce que les séquences de «Lance et compte» sont axées sur le système du reaction shot et que ce système puise dans le documentaire. On peut se demander si cette argumentation est suffisante pour expliquer la popularité de la série. Le montage ultra-nerveux, comme le souligne Gilles Marsolais ${ }^{3}$, contribue probablement à aiguiser tout autrement l'engouement des spectateurs. De même, ce détour par la série télévisuelle permet à l'auteur d'affirmer que «notre cinéma, dans sa structure narrative de base, est beaucoup plus américanisé qu'on le croit». Malgré l'intérêt d'une telle comparaison, on peut se demander si la série télévisuelle peut être analysée sur le même pied qu'une production cinématographique, car les conditions de production, de réalisation et de réception sont sensiblement différentes.

Malgré les dérives de l'auteur, cet ouvrage-synthèse nous conduit step by step et irrémédiablement au centre de la structure narrative hollywoodienne et nous ramène toujours à sa structure de base: le système du reaction shot qui se déploie en dernière instance selon un modèle vertical, à savoir l'axe écran/salle. Le but déguisé de la cinématographie populaire américaine est ni plus ni moins de faire entrer le spectateur, la salle obscure dans l'écran scintillant, sur la pellicule.

De Brando à Rambo, du ring à la patinoire, de Reagan à Swaggart, de Marilyn à Nancy (Reagan), on ne peut que conclure sur la fascination de Paul Warren à l'égard de la structure réactionnaire du reaction shot. Si l'on peut s'interroger sur le fait que l'auteur place divers systèmes de représentation selon un même régime de contiguïtés, Le Secret du star system américain nous oblige très certainement à envisager et à penser autrement le paysage audio-visuel.

Denise Pérusse

Université de Montréal

\section{NOTES}

1 Voir à ce sujet l'analyse percutante d'Ingrid Carlander, «La foire aux miracles des télévangélistes américains», Le Monde diplomatique (Paris, juin 1988), p. 12.

2 On n'admire pas Dieu; on croit en lui. Par contre, on peut à la fois croire et admirer Jimmy Swaggart. Ce que l'Église électronique perd en authenticité, elle le gagne en cote d'écoute.

3 Voir la critique de Gilles Marsolais dans 24 images ( $n^{\circ} 47$, janvier-février 1990), p. 90. 


\section{OUVRAGE CITÉ}

Postman, Neil. Se distraire à en mourir. Paris: Flammarion, 1986.

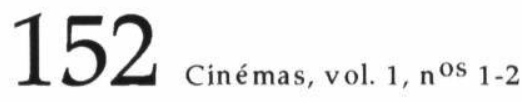

